\title{
STUDY ON ESTABLISHMENT SOYBEAN CONTROLLABLE STRUCTURAL MODEL
}

\author{
Zhongbin Su ${ }^{1,2, *}$ Ping Zheng ${ }^{2}$, Hongmin Sun $^{2}$, Jicheng Zhang $^{2}$, \\ Xiaoming $\mathrm{Li}^{2}$ \\ 1 Computer Science and Technology Academy Department, Beijing Institute of Technology, \\ Beijing, China, 100081 \\ 2 Engineering Academy, Northeast Agricultural University, Harbin, China, 150030 \\ * Corresponding author, Address: Computer Science and Technology Academy Department, \\ Beijing Institute of Technology, Beijing, 100081, P. R. China, Tel: +86-0451-55190170, \\ Fax: +86-0451-55190170, Email: suzb001@163.com
}

Abstract: By analyzing soybean structural and physiological characteristic sufficiently, the paper proposes a plant structural controllable modeling method for soybean. It selects the key structural factors firstly, and collects the relevant field data. Then it extracts the organ structure and position laws along with their vertical and horizontal change to establish controllable structural model. Combined with structural growth model based on growth function, it could establish soybean dynamic structural model, which has great significance to optimize the field cultivation measure and design ideal plant type.

Keywords: $\quad$ soybean; structural model; controllable model; dynamic model; visualization

\section{INTRODUCTION}

Virtual plant is one of the hot and difficult issues in digital agriculture research, which need to be solved urgently. It could study complex agricultural ecosystem directly and discover the plant principles that are observed difficultly by traditional research methods. It is helpful to understand thoroughly the crop structural and physiological law, optimize field cultivation measure, and design ideal plant type (Cao, 2005). Virtual plant also has vital significance to increase crops yield. At present, main research theories for virtual plant are L-system, AMAP model, particle system and three dimension reconstructions and ect. (D. Barthelemy, 2003).

Su, Z., Zheng, P., Sun, H., Zhang, J. and Li, X., 2008, in IFIP International Federation for Information Processing, Volume 258; Computer and Computing Technologies in Agriculture, Vol. 1; Daoliang Li; (Boston: Springer), pp. 685-693. 
These theories for modeling have their own value. Each one is suitable to simulate specific plant type. They all have certain application scales and some limitation (Guo, 2007).

Soybean is an important food and economic crop. Its growth simulation modeling research is quite mature. Typical example is American soybean growth simulation modeling, named "Soygro", which is used to forecast soybean growth developmental state, yield and simulate the balance of soil's moisture. The US has developed three decision support systems for soybean growth simulation modeling named "Soybean dss86" and "Pcyield" on the basis of "soygro" (Wang, 1982). But at present the soybean structural simulation research is relatively less. S. Chuai-Aree et al. developed "plantVR" based on L-system and growth equation technology. The simulation result of soybean structure is quite vivid, but this software considered less in environmental factors and community growth. So it has little instruction significance to soybean production. By analyzing above questions, this paper proposed one kind of controllable structural modeling method on the basis of understanding soybean structural and physiological characteristic. The structural model established by this method could flexibly display vertical distribution state of leaves and other organs. And it also expresses the trifoliolate leaf change in plant community. It provides a new research technique for establishing structural model interactive with environment, optimizing field cultivation measure, and designing ideal plant type (Su, 2005).

\section{MATIERIALS AND METHOD}

\subsection{Data measurement}

Field measurements were conducted at Xiangfang experimental field farm of Northeast Agricultural University from 2006 to 2007. Soybean cultivars Dongnong42 seed was sown $0.2 \mathrm{~m}$ apart in east-west-oriented rows that were $0.1 \mathrm{~m}$ apart. The result plant population is higher than that commonly used by local farmers and was chosen to maximize competition among around plants, the aim here being to analyze the plant structure with no branching. The plots were irrigated and fertilizer inputs were such as to avoid any mineral and water limitation to plant growth. There was one plant in a hole avoiding competition among one hole's plants. No plant disease, pest symptoms were observed.

The soybean plant has many structural characteristics, like alternate phyllotaxis, trifoliolate leaves, network vein and so on. So it took the 
relevant plans for the data collection. Plant structural data had been collected since the period of cotyledon. Each week 8 plants were taken randomly back to the laboratory for measuring. The collection data included fresh weight, length and diameter of each internode, fresh weight and area of each leaf; the angles between petioles and main stem; the angle between trifoliolate leaves and petiole.

Fresh weight was taken by electronic balance with accuracy $0.01 \mathrm{~g}$. The leaf area was measured by CI-203 Portable Laser Area Meter of CID Corporation with accuracy $0.001 \mathrm{~cm} 2$ and error $\pm 1 \%$. The length of internode and petiole was taken by straightedge with accuracy $0.1 \mathrm{~cm}$. The diameter of internode and petiole was taken by vernier caliper with accuracy $0.001 \mathrm{~cm}$. The angles were taken by protractor with accuracy $0.01^{\circ}$

\subsection{Controllable modeling method}

Plant structural model includes static model and dynamic model. Static model is established by plant structural data measured by three-dimensional digital methods. The model of this method is established directly by these data. It is only used to research the condition and state about plant space structure. Its drawback is transferring a great deal of data collected directly. It is not suitable to reflect plant structural dynamic characteristics. Dynamic model is established based on plant topology and geometry change laws. It is established by extracting plant growth laws. It is the main research direction of virtual plant modeling.

In the process of the establishing soybean structural dynamic model, the paper divides dynamic model into growth model and controllable model, which respectively manifests the growth process with time elapse and growth process with environmental change. In the process of establishing soybean growth model, it generally used growth equation. For example, Logistic equation is often used to simulate internode growth. In the previous simulation study, it has proven that the growth equation can effectively simulate soybean growth with time elapse.

In the process of establishing soybean controllable model, the influent factors considered are more complicated. It should take into account the number and position of plant organs on vertical and horizontal change and organ physiological change law affected by the external environment. The controlled model is established by extracting these factors' laws on the shape of organs, location, biomass and etc. The model could control the plant growth structure change and build plant physiological and ecological development process more really. 
3. RESULTS AND ANALYSIS

\subsection{Main stem and petiole}

\subsubsection{Growth model based on L-system}

The topological structure of "Dongnong 42" has strongly regularity that trifoliolate leaves petioles with same structure alternately grow on main stem. Three small leaves and petioles respectively compose a petiole region. On comparative analysis of existing model construction theories, the paper selects L-system to establish soybean topological model. First, it gives some symbols with special meanings in L-system, as shown in table 1 (Zheng, 2006).

Table 1. Some symbols' meanings in L-system

\begin{tabular}{clcl}
\hline Symbol & Meanings & Symbol & Meanings \\
\hline I & Length of internodes & P & Leafstalk \\
i & Litter leafstalk & L & Trifoliolate Leaves \\
P 1 & Left branch & L & Simple leaf \\
P r & Right branch & A & Growth point \\
P & Trifoliolate Leaves Region & Angle & Intersect angles \\
\hline
\end{tabular}

Through many experimental comparisons and observations, this paper designs topological qualitative model in L-system below:

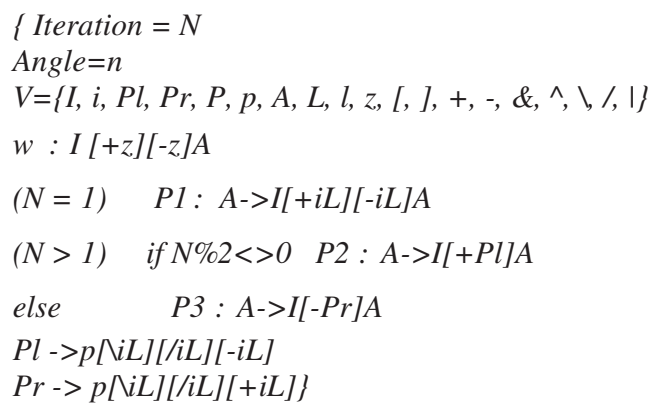

The amount of variable "Iteration" is conformed by days of soybean growth and partition of physiological period. For example, if given 126 days, "Iteration" equals 18, and it will create a new character string:

$$
\mathrm{I}[+\mathrm{Pr}] \mathrm{I}[-\mathrm{Pl}] \mathrm{I}[+\mathrm{Pr}] \mathrm{I}[-\mathrm{Pl}] \mathrm{I}[+\mathrm{Pr}] \mathrm{I}[-\mathrm{Pl}] \mathrm{I}[+\mathrm{Pr}] \mathrm{I}[-\mathrm{Pl}] \mathrm{I}[+\mathrm{Pr}] \mathrm{I}[-
$$
$\mathrm{Pl}] \mathrm{I}[+\mathrm{Pr}] \mathrm{I}[-\mathrm{Pl}] \mathrm{I}[+\mathrm{Pr}] \mathrm{I}[-\mathrm{Pl}] \mathrm{I}[+\mathrm{Pr}] \mathrm{A}$.

Now the letters in production (1) are expressed by turtle interpret. It can obtain static structure of "Dongnong 42" at about 120 days. Then it builds 
growth equations by collecting and analyzing the data of internode length and petiole etc. in the production (1). This method could establish growth model with time elapse.

\subsubsection{Controllable model simulation}

The samples of plants are measured every one-week. It observed and recorded the length of internode, the petiole of trifoliolate leaves, and the angles between main stem and petioles. This paper adopts angle data (as shown in table 2) to analysis and explanation. Matlab analyzes the collect data and finds that the numerical of the angles between main stem and petioles are similar to a quadratic curve by vertical distribution. This research did parameter fitting with Matlab, and obtained regression equation as shown in expression 2 .

$$
\begin{array}{ll}
y=0.0212 x^{2}-0.2036 x+0.9678 & 1 \leq x \leq 12 \\
y=-0.5236 x+7.854 & 12<x \leq 14 \\
y=0.5236 & x=15
\end{array}
$$

where $\mathrm{x}$ is the phyllotaxis between main stem and trifoliolate leave, and $\mathrm{y}$ is angle degree.

Table 2. The angle data between main stem and petiole

\begin{tabular}{lcccccccccc}
\hline Phyllotaxis & 1 & 2 & 3 & 4 & 5 & 6 & 7 & $\ldots \ldots$ & 13 & 14 \\
Angle & 0.80 & 0.64 & 0.55 & 0.50 & 0.50 & 0.55 & 0.62 & $\ldots \ldots$ & 1.04 & 0.5 \\
\hline
\end{tabular}

The units for angle are radian.

By this method, the paper extracts every soybean structural data with phyllotaxis to establish controllable model. The controllable model is expressed in delphi 7.0 on OpenGL to obtain the topological structure simulating results of "Dongnong 42". Fig. 1 showed three main stem type of soybean by this method. It can fully reflect relationship of the angles between main stem and petiole along with vertical direction change. It has great significance to further design the ideal plant type.

Soybean is field crop. Its inter plant distances and row distances are narrow. In the growth process it has more competition with surrounding plants. That is to say, the plant structure is affected by plant cultivation
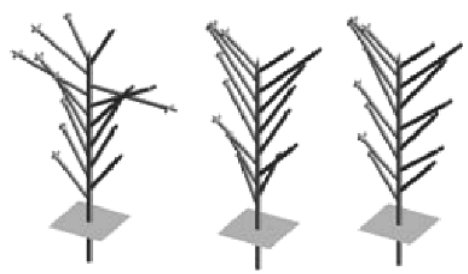

Fig. 1. Petiol's azimuth vertical change simulation 
position and plant density. The symbol "+" and "-" in equation (2) are expressed space angles between main stem and petioles. The paper changes them by analyzing the growth state of the surrounding plants state. Then it extracts the laws of horizontal angels' laws and plant's location. The method could simulate the horizontal change law of soybean structure. It is helpful to simulate soybean community growth, as shown in Figure 2.

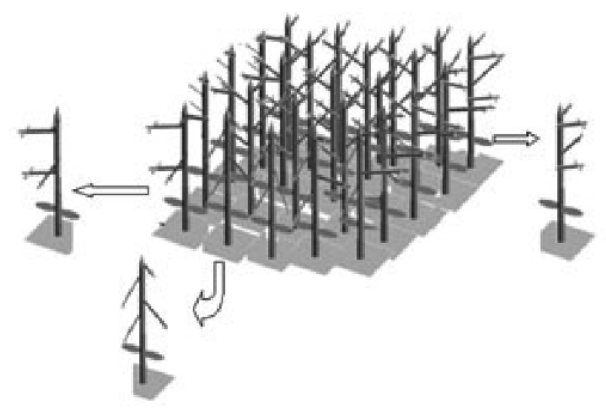

Fig. 2. Petiole's azimuth horizontal change simulation

\subsection{Trifoliolate leaf}

Crop production is related with the photosynthesis closely. It could guarantee high and stable production by selecting cultivars with high photosynthetic efficiency (HPE) to improve the absorption intensity of light energy and taking reasonable field cultivation measure. Soybean leaf is an important organ to carry on the photosynthesis and make the organic nourishment. The simulating study thoroughly of leaf can provide basis and methods for designing plants type and selecting cultivars. According to soybean leaf' features, such as alternate phyllotaxis, trifoliolate leaf, network veins and so on, the paper selects suitable methods to establish growth models and controllable models to increase 3D effect and realistic feeling. It could provide methods to establish more reasonable plant structure and design ideal plant type (Kang, 2006).

\subsubsection{Leaf outline}

In the previous study, leaf outline could be obtained by a series of digital processing that is graying, extracting outline, vectorization in VC platform. This method can reflect leaf's static condition, but it lacks the description of the leaf growth. The paper establishes leaf outline by growth function and Bezier curve. It could simulate the leaf growth process with high speed and efficiency. 
First, collect the length and width data of each trifolioate leaf, as shown in Table 3.

Table 3. Data of leaf's Length and Width

\begin{tabular}{lccccrrr}
\hline Time & 1 & 3 & 6 & 9 & 13 & 15 & 18 \\
\hline Width & 0.30 & 1.00 & 1.50 & 2.70 & 4.80 & 5.90 & 6.10 \\
Length & 0.50 & 1.80 & 3.50 & 5.80 & 10.50 & 11.70 & 12.00 \\
\hline
\end{tabular}

The units for Time are day; Width is $\mathrm{cm}$; Length is $\mathrm{cm}$.

Then, the data is regressed by Richards equation, as shown in equation (3),

$$
\begin{aligned}
& P_{1}(y)=\frac{6.02299}{\left(1+10799.224 e^{-0.6327515}\right)^{1 / 399}} \\
& P_{2}(y)=\frac{12.1673}{\left(1+1748.59 e^{-0.6277 \Omega}\right)^{1 / 2.76}}
\end{aligned}
$$

Select the leaf petiole nod " $\mathrm{p}_{0}$ " as the origin of coordinates, and regard " $p_{1}$ "and " $p_{2}$ " as apexes of the leaf"s width and length, as shown in Fig. 3 (a). Line segments " $\mathrm{p}_{2} \mathrm{p}_{4}$ " and " $\mathrm{p}_{4} \mathrm{p}_{5}$ " respectively express tangents, and " $\mathrm{p}_{2}$ " and " $p_{1}$ " express the tangential point. "p" expresses a random point in the curve " $\mathrm{p}_{0} \mathrm{p}_{1}$ ". It introduces a parameter " $\mathrm{k}$ ". According to De Casteljau algorithm it could get the equation (4).

$$
\mathrm{P}=(1-k)^{2} \mathrm{P}_{0}+2 k(1-k) \mathrm{P}_{5}+k^{2} \mathrm{P}_{1}
$$

When $\mathrm{k}$ range from 0 to 1 , "P" expresses quadric Bezier curves named curve " $\mathrm{P}_{0} \mathrm{P}_{1}$ " which is defined by three apexes " $\mathrm{P}_{0}$ ", " $\mathrm{P}_{1}$ " and " $\mathrm{P}_{5}$ ". So as curve " $\mathrm{P}_{1} \mathrm{P}_{2}$ ". This method can draw left part of leaf outline through these steps, as shown in Fig. 3. Symmetrical rotating from the left part can draw the right part. This method could create leaf outline based on growth function and Bezier curves. It gets a smooth outline figure that describes leaf growth by limited iteration.

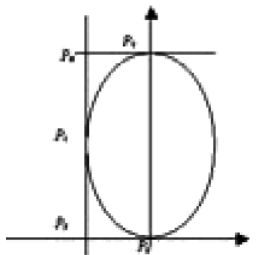

(a)

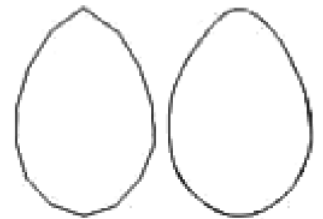

(c)

Fig. 3. Outline curve fitting process (a) outline's sketch; (b) outline drawn by 10 iteration; (c) outline drawn by 50 iteration 


\subsubsection{Controllable model simulation}

After chapter 2.1.2 research, it builds controllable model of main stem and petiole. It can simulate leaf petiole azimuth change along with phyllotaxis and plant location. Three leaves think as a whole because of trifoliolate leaf. Through observation and experiments measurement, left and right leaves' main veins and middle leaf identify a plane, named trifoliolate leaf plane as shown in Fig. 4 (a). The angle between the plane and petiole lies on trifoliolate leaf phyllotaxis and light source. The left and right leaves appropriately rotate with their main veins according with light intensity to increase or decreases energy absorption, as shown in Fig. 4 (b), (c). In the leaf controllable model establishment process, it selects parameters to express trifoliolate leaf plane and petiole, left and right leaves and this plane. Then extract the law about two angles along with phyllotaxis and light source to establish leaf structural model. The model considers more about leaf physiology characteristic to give prominence to organ of absorbing light. It could increase soybean structural model's flexibility.

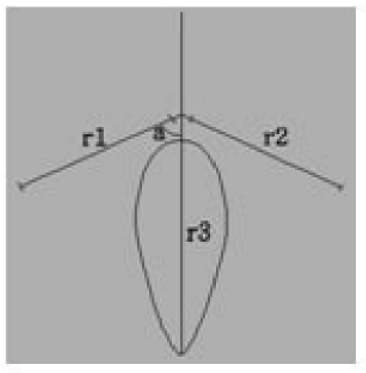

(a)

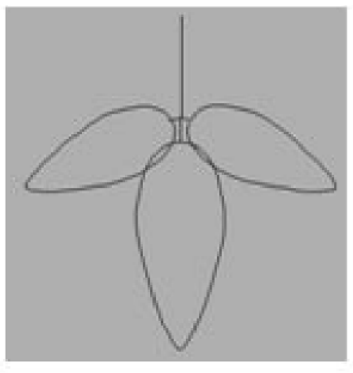

(b)

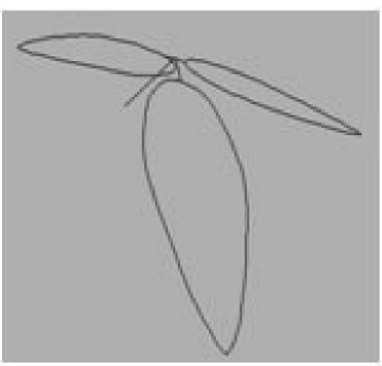

(c)

Fig. 4. Controllable leaf structural model (a) trifoliolate leaf plane; (b) left and right leaves in the plane; (c) some angles degree between two leaves and plane

\section{CONCLUSION}

The paper establishes a soybean controllable structural model by analyzing and simulation the angles between main stem and petiole, trifoliolate leaf and petiole and some key factors. The model implements preliminary structural influence on plant location and light source from environmental factors. The model accords with soybean structural growth laws better. It has positive effect in establishment interactive model with external environmental factors. In further study, the model need combine with soybean physiology to strengthen further analysis of structural collection data. It would be helpful to extract the laws between plant location 
and structure, environmental factors and structure, yield and structure to establish more realistic soybean structural model.

\section{ACKNOWLEDGEMENTS}

This study has been funded by Chinese 863 Plan (\#2006AA10Z231), Heilongjiang Natural Science Foundation of China (\#C200607), and Program for Innovative Research Team of Northeast Agricultural University, "IRTNEAU".

\section{REFERENCES}

Cao Wei-xing. Agricultural Information Science. Bei Jing: China Agriculture Publishing, 2004. (in Chinese)

D. Barthelemy. Botanical Background for Plant Architecture Analysis and Modeling. In: Plant Growth Modeling and Applications Proceedings. Beijing: Tsinghua University Press. 2003:1-20

F. Normand, J. Chadoeuf, R. Habib. Modeling Asynchronous Flowering. In: Plant Growth Modeling and Applications Proceedings. Beijing: Tsinghua University Press. 2003:76-84

Guo Xinyu, Zhao Chunjiang, Xiao Boxiang, et al. Design and implementation of threedimensional geometric morphological modeling and visualization system formaize. Journal of Agricultural Engineering. 2007, 4, 144-149. (in Chinese)

Kang Li, Su Zhong-bin, Zheng Ping, et al. Research on Modeling Leaf Based on L-system, Agricultural Machinery Research, 2006, 7, 180-182. (in Chinese)

Song Yonghong, Guo Yan, Li Baoguo, et al. Virtual maize model .plant morphological constructing based on Organ biomass accumulation. Ecological Journal. 2003, 23(12): 2579-2586. (in Chinese)

$\mathrm{Su}$ Zhongbin, Meng Fanjiang, Kang Li, et al. Virtual plant modeling based on Agent technology. Agricultural Engineering. 2005, 21 (8):114-116. (in Chinese)

Wang Jingwen, Yi Tianfu. Soybean plant type mathematical models and canopy light of the vertical distribution. Journal of Northeast Agriculture. 1982, 3, 1-9. (in Chinese)

Zheng Ping, Su Zhongbin, Kang Li. Modeling of Virtual Soybean Topology Based on Growth Function, Agricultural Machinery Research, 2006, 7, 193-194. (in Chinese) 